\title{
Effects of high thoracic epidural anesthesia on ischemic cardiomyopathy cardiac function and autonomic neural function
}

\author{
X. Wang ${ }^{1 *}$, G.Y. Chen ${ }^{1 *}$, S.S. Yang ${ }^{1}$, Y. Tian ${ }^{1}$, T. Ge ${ }^{2}$, H. Qin ${ }^{2}$, W. Han ${ }^{1}$ \\ and H.Y. Chang ${ }^{1}$ \\ ${ }^{1}$ Department of Cardiology, \\ The First Affiliated Hospital of Harbin Medical University, \\ Nangang District, Harbin, Heilongjiang, China \\ ${ }^{2}$ Department of Medical, Second Hospital of Harbin City, Harbin, \\ Heilongjiang, China \\ *These authors contributed equally to this study. \\ Corresponding authors: S.S. Yang / Y. Tian \\ E-mail: yangshusen@medmail.com / yetian6@163.com
}

Genet. Mol. Res. 13 (3): 6813-6819 (2014)

Received June 10, 2013

Accepted October 31, 2013

Published August 29, 2014

DOI http://dx.doi.org/10.4238/2014.August.29.2

\begin{abstract}
We aimed at observing the effects of high thoracic epidural anesthesia (HTEA) on cardiac structure and function, heart rate variability (HRV), and QT interval variation (QTV) in ischemic cardiomyopathy (ICM) patients with chronic heart failure. We divided 30 ICM patients into HTEA $(\mathrm{N}=16)$ and control $(\mathrm{N}=14)$ groups. The control group was treated with medication, and the HTEA group was treated with HTEA and medication for 4 weeks. We measured the changes in the left-ventricular end-diastolic diameter (LVEDd) and left-ventricular ejection fraction (LVEF) before and after treatment by using echocardiography and examined changes in HRV and QTV using ambulatory electrocardiogram. HTEA significantly narrowed the LVEDd, improved LVEF, significantly increased the HRV, and significantly reduced the QTV in the ICM group compared to the control group. HTEA significantly narrowed the
\end{abstract}


ventricular chamber diameter size of ICM patients, enhanced myocardial contractility, increased myocardial electrical stability, and improved the cardiac structure and function.

Key words: High thoracic epidural anesthesia; Heart rate variability; Ischemic cardiomyopathy; Chronic heart failure

\section{INTRODUCTION}

Chronic heart failure (CHF) is a complex clinical syndrome manifested at the end stage of a variety of serious heart disease. Although the incidence of other cardiovascular diseases has decreased annually, the incidence of heart failure shows an increasing trend, and this increase may be associated with the aging of the population (Anversa and Nadal-Ginard, 2002). Therefore, the study of the mechanism of heart failure development has been one of the priorities in the global prevention and control of cardiovascular disease. In CHF, the activation of the sympathetic adrenaline nervous system, the renin-angiotensin system, and other endocrine systems causes cardiac hypertrophy, myocardial fibrosis, and myocardial apoptosis, which promote ventricular remodeling. Greenberg (2001) pointed out that until the 1990s, the decisive mechanism of heart failure was myocardial remodeling.

The heart is dominated by sympathetic fibers from the upper thoracic spinal cord (T1-5). Heart rate mainly depends on the balance between the sympathetic and parasympathetic systems. High thoracic epidural anesthesia (HTEA) involves 5 thoracic nerve segments, blocking the incoming and outgoing sympathetic fibers that dominate the heart and resulting in the loss of myocardial chronotropic and inotropic driving force. When sympathetic fibers cannot dominate the heart due to HTEA, parasympathetic fibers take over the cardiovascular response. Therefore, the heart rate decreases after treatment with HTEA (Veering and Cousins, 2000), no significant changes occur in cardiac output (but a significant decrease in systemic vascular resistance was observed), and the skin blood flow increases (Nygård et al., 2002). At the same time, the peripheral local blood supply and oxygen supply improves (Buggy et al., 2002; Kabon et al., 2003; Treschan et al., 2003). HTEA can effectively control tachycardia by selectively blocking sympathetic fibers (T1-5) that dominate the heart and reduce thrombotic complications, relieve angina, reduce cardiac stunning, improve left ventricular function, and improve myocardial oxygen supply and demand balance. This effect is particularly evident in ischemic cardiomyopathy (ICM).

In this study, we examined the effects of HTEA on cardiac structure and function, heart rate variability, and QT interval variability (QTV) in ICM patients with chronic heart failure.

\section{MATERIAL AND METHODS}

\section{Patients}

We collected data of 30 patients with ICM who were hospitalized between October 2005 to October 2006. The patients were divided to 2 groups: HTEA group ( $\mathrm{N}=16 ; 12 \mathrm{men}$; age, $54 \pm 18$ years) and control group $(\mathrm{N}=14 ; 10$ men; age, $55 \pm 17$ years). The criteria of 
ICM were as follows: clear old myocardial infarction medical history, significantly expanded heart, meeting the diagnostic criteria of chronic heart failure, LVEF $<40 \%$, New York Heart Association functional class $\beta-\varepsilon$ grade. Exclusion criteria included mechanical complications of coronary heart disease (ventricular septal rupture, papillary muscle dysfunction) and other heart diseases (primary cardiomyopathy, rheumatic heart disease, hypertension, chronic anemia, hyperthyroidism, etc.) caused by heart expansion, heart failure, and renal insufficiency.

Patients in the control group were treated with medications, including sodium nitroprusside, dopamine, Cedilanid, and furosemide for a course of 4 weeks. Patients in the HTEA group were treated with HTEA and drainage as well as medical treatment.

\section{High thoracic epidural anesthesia}

Patients were asked to lay in the lateral recumbent position or sitting position. A thoracicepidural catheter was inserted at the T4-T5 interspace using the loss-of-resistance technique, and then advanced 4 to $5 \mathrm{~cm}$ cephalad after it was placed in the T1-T2 interspaces. The temperature at the anesthesia area was recorded using a skin thermometer. In these regions, the temperature may rise by 0.5 to $1.0^{\circ} \mathrm{C}$. A sufficient amount of lidocaine $(0.5 \%)$, usually 3 to $5 \mathrm{~mL}$ was given epidurally to induce blockade of cardiac sympathetic segments (T1 to T5). The patients were injected with 3 to $5 \mathrm{~mL} 0.5 \%$ lidocaine every 2 hours for 4 weeks. The signs of successful blockage included miosis, conjunctival hyperemia, ptosis, and skin temperature increase.

\section{Indicators and methods}

\section{Echocardiography}

All patients underwent echocardiography (ECG) (VIVID FIVE; probe frequency of $2.5 \mathrm{MHz}$; GE, USA) before and 4 weeks after treatment. Patients lay in the lateral recumbent position or left lateral decubitus position, and the probe was placed in the parasternal long axis view. We measured the left atrial systolic diameter (Lad) at end diastole using M-mode ultrasound, left ventricular end-diastolic diameter (LVEDd), and left ventricular fractional shortening (LVFS). In the apical four-chamber view, we measured the left ventricular end-diastolic and end-systolic volume (LVEDV, LVESV) using the area-length method and calculated the left ventricular end-diastolic and end-systolic volume (LVEDV, LVESV) and left ventricular ejection fraction $[\mathrm{LVEF}=(\mathrm{LVEDV}-\mathrm{LVESV}) / \mathrm{LVEDV}]$ using the area-length method. Each indicator was measured three times and the average value was used. All examinations were performed by the same experienced examiner.

\section{Holter monitoring}

Before and 4 weeks after treatment, all the selected patients underwent 24-h ambulatory ECG. We analyzed the data using the CENTURY SERIES Biomedical Systems V1.30 Holter software. Effective dynamic ECG was used to monitor sinus rhythm, except of seconddegree or above sinoatrial, atrioventricular, or bundle branch block. The ectopic beats were filtered and interference was record for more than 22 hours by effective ECG.

Parameters used were standard deviation (SDNN) of all sinus RR during the detection 
intervals. The average standard deviation (SDANN) of segments' sinus RR at every 5-min interval during the entire process. QTV, which dynamically measured the QT interval of the same lead by wave and calculated the standard deviation of the corresponding segments of the QTV.

\section{Statistical analysis}

SPSS12.0 was used for statistical analysis. Measurement data are reported as means \pm standard deviation. Data between two groups were compared using the independent sample $t$-test. For each patient, we compared the differences before and after treatment by using the paired $t$-test. The differences were considered to be statistically significant when $\mathrm{P}<0.005$.

\section{RESULTS}

\section{Echocardiography}

Before and 4 weeks after treatment, the two groups of patients were examined using ECG. Lad, LVEDd, LVEF, and LVFS were not significantly different $(\mathrm{P}>0.05)$ between the two groups before treatment. Lad and LVEDd significantly decreased and LVEF and LVFS showed a significant increase $(\mathrm{P}<0.01)$ after treatment the HTEA group. In the control group, LVFS decreased significantly $(\mathrm{P}<0.05)$ after treatment and the Lad, LVEDd, and LVEF showed no significant difference $(\mathrm{P}>0.05)$.

Compared with the control group, LVEDd decreased significantly and LVEF and LVFS increased significantly in the HTEA group after treatment $(\mathrm{P}<0.01)$. See Table 1.

\begin{tabular}{|c|c|c|c|c|c|}
\hline Group & Patients $^{\#}$ & Lad (mm) & LVEDd (mm) & LVEF (\%) & LVFS (\%) \\
\hline HTEA group & 16 & & & & \\
\hline Before treatment & & $47.25 \pm 3.80$ & $67.88 \pm 3.40$ & $27.13 \pm 4.59$ & $12.94 \pm 2.41$ \\
\hline After treatment & & $44.25 \pm 3.15^{* *}$ & $59.69 \pm 3.79^{* * *}$ & $35.50 \pm 5.13^{* * *}$ & $16.94 \pm 2.26^{* *}$ \\
\hline Control group & 14 & & & & \\
\hline Before treatment & & $47.08 \pm 3.59$ & $66.39 \pm 5.91$ & $27.92 \pm 4.72$ & $12.69 \pm 1.75$ \\
\hline After treatment & & $46.31 \pm 2.72$ & $65.54 \pm 4.79$ & $29.77 \pm 4.25$ & $13.62 \pm 1.85^{*}$ \\
\hline
\end{tabular}

\section{Holter monitoring}

Before and 4 weeks after treatment, all selected patients underwent 24-hour ambulatory ECG for determination of SDNN, SDANN, and QTV. The SDNN, SDANN, and QTV did not differ significantly between the two groups before treatment $(\mathrm{P}>0.05)$. However, compared with before treatment, SDNN and SDANN increased significantly $(\mathrm{P}=0.002$ and 0.001 respectively) and QTV decreased significantly $(\mathrm{P}=0.006)$ after treatment in the HTEA group. In the control group, SDNN and SDANN increased significantly $(\mathrm{P}=0.001$ and 0.0029 , respectively), and QTV decreased significantly $(\mathrm{P}=0.046)$, but no such change was evident in the HTEA group.

Compared with the control group, after treatment, SDNN and SDANN increased significantly in the HTEA group and QTV reduced significantly $(\mathrm{P}<0.05)$, as shown in Table 2. 


\begin{tabular}{|c|c|c|c|c|}
\hline Group & Patients $^{\#}$ & SDNN (ms) & SDANN (ms) & QTV (ms) \\
\hline HTEA group & 16 & & & \\
\hline Before treatment & & $97.57 \pm 12.86$ & $80.43 \pm 15.92$ & $24.57 \pm 6.54$ \\
\hline After treatment & & $112.43 \pm 18.30^{* * \#}$ & $98.43 \pm 8.89 * * \#$ & $18.43 \pm 2.14 * * \#$ \\
\hline Control group & 14 & & & \\
\hline Before treatment & & $92.25 \pm 13.43$ & $81.75 \pm 17.02$ & $28.83 \pm 4.24$ \\
\hline After treatment & & $99.92 \pm 14.39^{*}$ & $85.75 \pm 15.78^{*}$ & $24.67 \pm 3.58^{*}$ \\
\hline
\end{tabular}

\section{DISCUSSION}

ICM is a severe myocardial dysfunction caused by chronic myocardial ischemia after coronary artery stenosis or occlusion, accompanied by multifocal ventricular wall motion abnormalities (Rodkey et al., 1998). The progression of ICM to heart failure was caused by myocardial infarction, myocardial fibrosis, and myocardial hibernation, clinically manifested as progressive enlargement of the heart, cardiac dysfunction, thromboembolism, arrhythmias, and sudden death. Various conditions lead to repeated deterioration of heart function, for which the routine medical anti-heart failure medication is less effective, and currently, there is no fundamental solution for this problem except for heart transplantation.

Cardiac sympathetic activation was the earliest and most strongly during the heart failure. Cardiac sympathetic overactivity can damage myocardial cells by causing a release of large amount of catecholamines and cytokines. Therefore, locally blocking cardiac sympathetic fibers could considerably protect the heart and reduce the secondary effects. Domination of the heart by the sympathetic lateral horn of the thoracic spinal cord (T1-5) through the sympathetic ganglion preganglionic fibers changes the postganglionic fibers. Cardiac sympathetic overactivity can be completely inhibited, and the benefit would increase if the proximal sympathetic fiber was blocked. HTEA can confer these effects. Recently, Nygård et al. (2005) confirmed that HTEA could block cardiac sympathetic overactivity and ensure a partly normal myocardial blood flow in ICM patients with multivessel coronary disease. Myocardial blood flow is essential for protection of heart function during percutaneous coronary intervention (PCI). HTEA directly blocks the sympathetic preganglionic fiber conduction, which dominates the heart, thereby reducing the release of catecholamines by postganglionic fibers and inhabiting excessive activation of the sympathetic nervous system, leading to a good therapeutic effect in patients with severe heart failure (Wu et al., 2007).

In this study, we examined the effect of HTEA on heart function, heart rate variability, and QTV in ICM patients with chronic heart failure. Ventricular remodeling is an important mechanism in the development and progression of heart failure (Takano et al., 2003). The results of this study showed that the HTEA could significantly narrow the LVEDd, increase the LVEF, and improve heart function in ICM patients, especially for those with severe ICM, those who were not suitable for PCI, and those with refractory heart failure awaiting a heart transplant. HTEA could delay ventricular remodeling in ICM patients by selectively blocking the cardiac sympathetic nerve, which could reduce the sympathetic activity in patients with heart failure, reduce the damage to the myocardium by catecholamines, expand the coronary artery (Loick et al., 1999), improve the ischemic myocardium oxygen supply, and balance and improve myocardial 
metabolism (Carli and Klubien, 1999). HTEA could significantly improve not only the contractile function and reduce the left ventricular cavity diameter of patients with severe heart failure, but also the diastolic function of left myocardial tissue as confirmed by tissue Doppler, which may be the basis for improving the prognosis of heart failure (Schmidt et al., 2005; Xiu et al., 2006).

Heart rate variability (HRV) has been widely used in monitoring a variety of cardiovascular diseases through changes in cardiac autonomic functional reaction and is an important indicator for auxiliary diagnostic and prognostic evaluation. In this study, we performed HRV analysis for 30 ICM patients with heart failure. Our results showed that SDNN and SDANN increased significantly after treatment in the HTEA group $(\mathrm{P}<0.01)$; although these parameters increased in the control group as well, $(\mathrm{P}<0.05)$, the difference was not as significant as that in the HTEA group. Compared with the control group, the SDNN and SDANN were significantly different in HTEA group after treatment. The mechanism involved in increasing HRV may include the inhibition of the sympathetic nervous system activity, blockade of neurohormonal activation, reduction in the heart rate, reduction in myocardial oxygen consumption, and improvement in myocardial compliance. The HTEA improved cardiac electrical stability and ventricular fibrillation threshold and reduced the occurrence of malignant arrhythmias by increasing the HRV. Animal experiments have demonstrated the protective mechanism of HTEA in anti-ventricular and atrial arrhythmias. In a canine model, through prolonging repolarization and refractory period, HTEA reduced cardiac sympathetic activity, such as protective effect to ventricular tachycardia due to increased sympathetic tone (Meissner et al., 2001). In another experiment in rabbits, HTEA significantly reduced enflurane-/ epinephrine-induced arrhythmias.

Studies showed that in atrial fibrillation in dogs, HTEA inhibited the electrical and nerve remodeling of atrial fibrillation and played a role as an anti-inflammatory and antioxidant factor (Lee et al., 1999; Yang et al., 2011).

In ICM patients with chronic heart failure, myocardial injury or ischemia significantly changes the ventricular repolarization (Piccirillo et al., 2002a), consequently hampering timely depolarization and repolarization according to the needs of the body and complete conversion between electrical activity and mechanical activity. Therefore, in clinical practice, electrophysiological disorders are closely linked to arrhythmia and cardiac insufficiency (Piccirillo et al., 2002b). QTV, the mean standard deviation of hourly QT interval, dynamically reflects the ventricular repolarization changes. QTV is a good indicator of non-invasive detection of ventricular repolarization dispersion. From a statistical viewpoint, the calculation of this variability is more reliable than that of the range value (QT dispersion), and QTV is measured from the same lead axis, while the start site and termination sites of QRS-T waveform are relatively fixed. Therefore, QTV could be accurately measured with a good repeatability. Ventricular repolarization dispersion could be dynamically noted at any period. In this study, we performed QTV analysis for the 30 ICM patients with chronic heart failure, and the results showed that QTV was significantly decreased in both HTEA and control groups after treatment, but this difference was not as evident in the HTEA group as it was in the control group. Compared with the control group, in the HTEA group, QTV decreased significantly after treatment. HTEA reduced the difference in ventricular muscle repolarization, thereby reducing the incidence of arrhythmias, particularly ventricular arrhythmias. HTEA increased the stability of ventricular repolarization and improved ventricular repolarization imbalance by reducing the heart rate.

The sample size of this study was relatively small; therefore, the exact methods of 
treatment of ICM in patients with chronic heart failure need to be examined further.

\section{ACKNOWLEDGMENTS}

Research supported by the Science and Technology Research Project by Education Department of Heilongjiang Province (\#11541216) and the Scientific Research Project by Health Department of Heilongjiang Province (\#2009-052)

\section{REFERENCES}

Anversa P and Nadal-Ginard B (2002). Myocyte renewal and ventricular remodelling. Nature 415: 240-243.

Buggy DJ, Doherty WL, Hart EM and Pallett EJ (2002). Postoperative wound oxygen tension with epidural or intravenous analgesia: a prospective, randomized, single-blind clinical trial. Anesthesiology 97: 952-958.

Carli F and Klubien K (1999). Thoracic epidurals: is analgesia all we want? Can. J. Anaesth. 46: 409-414.

Greenberg B (2001). Treatment of heart failure: state of the art and prospectives. J. Cardiovasc. Pharmacol. 38 (Suppl. 2): S59-S63.

Kabon B, Fleischmann E, Treschan T, Taguchi A, et al. (2003). Thoracic epidural anesthesia increases tissue oxygenation during major abdominal surgery. Anesth. Analg. 97: 1812-1817.

Lee SC, Lee C and Kim YC (1999). Epinephrine-induced arrhythmias: effects of thoracic epidural anesthesia and vagotomy during enflurane anesthesia in rabbits. J. Korean Med. Sci. 14: 133-137.

Loick HM, Schmidt C, Van Aken H, Junker R, et al. (1999). High thoracic epidural anesthesia, but not clonidine, attenuates the perioperative stress response via sympatholysis and reduces the release of troponin $\mathrm{T}$ in patients undergoing coronary artery bypass grafting. Anesth. Analg. 88: 701-709.

Meissner A, Eckardt L, Kirchhof P, Weber T, et al. (2001). Effects of thoracic epidural anesthesia with and without autonomic nervous system blockade on cardiac monophasic action potentials and effective refractoriness in awake dogs. Anesthesiology 95: 132-138.

Nygård E, Sejrsen P and Kofoed KF (2002). Thoracic sympatholysis with epidural blockade assessed by quantitative measurement of cutaneous blood flow. Acta Anaesthesiol. Scand. 46: 1037-1041.

Nygård E, Kofoed KF, Freiberg J, Holm S, et al. (2005). Effects of high thoracic epidural analgesia on myocardial blood flow in patients with ischemic heart disease. Circulation 111: 2165-2170.

Piccirillo G, Nocco M, Lionetti M, Moise A, et al. (2002a). Effects of sildenafil citrate (Viagra) on cardiac repolarization and on autonomic control in subjects with chronic heart failure. Am. Heart J. 143: 703-710.

Piccirillo G, Germano G, Quaglione R, Nocco M, et al. (2002b). QT-interval variability and autonomic control in hypertensive subjects with left ventricular hypertrophy. Clin. Sci. 102: 363-371.

Rodkey S, Ratliff N and Young J (1998). Cardiomyopathy and Myocardial Failure. Lippincott-Raven Publishers, Philadelphia, 2589-2620.

Schmidt C, Hinder F, Van Aken H, Theilmeier G, et al. (2005). The effect of high thoracic epidural anesthesia on systolic and diastolic left ventricular function in patients with coronary artery disease. Anesth. Analg. 100: 1561-1569.

Takano H, Hasegawa H, Nagai T and Komuro I (2003). Implication of cardiac remodeling in heart failure: mechanisms and therapeutic strategies. Intern. Med. 42: 465-469.

Treschan TA, Taguchi A, Ali SZ, Sharma N, et al. (2003). The effects of epidural and general anesthesia on tissue oxygenation. Anesth. Analg. 96: 1553-7.

Veering BT and Cousins MJ (2000). Cardiovascular and pulmonary effects of epidural anaesthesia. Anaesth. Intensive Care 28: 620-635.

Wu S, Fu SY, Liu FQ, Wang LF, et al. (2007). Clinical observation of high thoracic epidural anesthesia therapy for patients with congestive heart failure secondary to ischemic cardiomyopathy. Zhonghua Yi Xue Za Zhi 87: 1752-1754.

Xiu CH, Liu FQ, Wang HQ, Wang X, et al. (2006). Effects of cardiac sympathetic blockade on left ventricular diastolic function in patients with dilated cardiomyopathy and severe heart failure. Zhonghua Yi Xue Za Zhi 86: 1170-1173.

Yang SS, Han W, Cao Y, Dong G, et al. (2011). Effects of high thoracic epidural anesthesia on atrial electrophysiological characteristics and sympathetic nerve sprouting in a canine model of atrial fibrillation. Basic Res. Cardiol. 106: 495506. 